\title{
Can the microRNA expression profile help to identify novel targets for zoledronic acid in breast cancer?
}

\author{
Daniele Fanale ${ }^{1, *}$, Valeria Amodeo ${ }^{1, *}$, Viviana Bazan ${ }^{1, *}$, Lavinia Insalaco ${ }^{1}$, Lorena \\ Incorvaia ${ }^{1}$, Nadia Barraco ${ }^{1}$, Marta Castigliaa ${ }^{1}$, Sergio Rizzo', Daniele Santini², \\ Antonio Giordano ${ }^{3}$, Sergio Castorina ${ }^{4,5}$, , , Antonio Russo ${ }^{1, \#}$ \\ ${ }^{1}$ Department of Surgical, Oncological and Oral Sciences, Section of Medical Oncology, University of Palermo, Palermo, Italy \\ ${ }^{2}$ University Campus Bio-Medico, Department of Medical Oncology, Rome, Italy \\ ${ }^{3}$ Sbarro Institute for Cancer Research and Molecular Medicine, Center for Biotechnology, College of Science and Technology, \\ Temple University, Philadelphia, PA, USA \\ ${ }^{4}$ Fondazione Mediterranea "G.B. Morgagni", Catania, Italy \\ ${ }^{5}$ Department of Biomedical and Biotechnological Sciences, University of Catania, Catania, Italy \\ \#Co-last authors, these authors contributed equally to this work \\ *These authors contributed equally to this work
}

Correspondence to: Antonio Russo, e-mail: antonio.russo@usa.net

Keywords: bone metastasis, breast cancer, microarray analysis, miRNA expression profile, zoledronic acid

Received: March 08, 2016

Accepted: March 31, 2016

Published: April 13, 2016

\section{ABSTRACT}

Zoledronic acid (ZOL), belonging to third generation bisphosphonate family, is a potent inhibitor of osteoclast-mediated bone resorption, widely used to effectively prevent osteolysis in breast cancer patients who develop bone metastases. Low doses of ZOL have been shown to exhibit a direct anticancer role, by inhibiting cell adhesion, invasion, cytoskeleton remodelling and proliferation in MCF-7 breast cancer cells. In order to identify the molecular mechanisms and signaling pathways underlying the anticancer activity exerted by $Z O L$, we analyzed for the first time the microRNA expression profile in breast cancer cells. A large-scale microarray analysis of 377 miRNAs was performed on MCF7 cells treated with $10 \mu \mathrm{M}$ ZOL for $24 \mathrm{~h}$ compared to untreated cells. Furthermore, the expression of specific ZOL-induced miRNAs was analyzed in MCF-7 and SkBr3 cells through Real-time PCR. Low-dose treatment with ZOL significantly altered expression of 54 miRNAs. Nine upregulated and twelve downregulated miRNAs have been identified after $24 \mathrm{~h}$ of treatment. Also, ZOL induced expression of 11 specific miRNAs and silenced expression of 22 miRNAs. MiRNA data analysis revealed the involvement of differentially expressed miRNAs in PI3K/Akt, MAPK, Wnt, TGF- $\beta$, Jak-STAT and mTOR signaling pathways, and regulation of actin cytoskeleton. Our results have been shown to be perfectly coherent with the recent findings reported in literature concerning changes in expression of some miRNAs involved in bone metastasis formation, progression, therapy resistance in breast cancer. In conclusion, this data supports the hypothesis that ZOL-induced modification of the miRNA expression profile contributes to the anticancer efficacy of this agent.

\section{INTRODUCTION}

Breast cancer $(\mathrm{BC})$ is the most common cancer and the major cause of cancer death in women worldwide $[1,2]$. The mortality associated to $\mathrm{BC}$ is correlated with bone metastasis in about $50 \%$ of patients, therefore, maintaining bone integrity is important for these patients [3]. Metastatic bone disease (MBD) is a painful complication characterized by elevated rates of localized osteolysis, which can lead to potentially debilitating skeletal-related events (SREs) such as pain, pathological fracture, hypercalcaemia and spinal cord compression $[4,5]$. Interference in the complex interactions between tumor and bone cells in the bone microenvironment results in MBD. Cytokines and growth factors produced by tumor cells destroy the balanced process between osteoclastic 
bone resorption and osteoblastic bone formation causing increased osteolysis. The alteration and disruption of the normal surrounding extracellular matrix (ECM), induced by matrix metalloproteinases (MMPs), determines the primary tumor cell invasion leading to development of bone metastasis $[6,7]$. This event causes the extravasation of cancer cells reaching distant target organs including skeletal tissue, where they establish respective metastases [8].

Bisphosphonates (BP) are potent inhibitors of osteoclast-mediated bone resorption approved for the treatment of MBD from advanced cancers, including $\mathrm{BC}$ [9]. Zoledronic acid (ZOL) is a third generation nitrogen-containing BP (N-BP) (Figure 1A) used for treatment of $\mathrm{BC}$ patients with osteolytic lesions in order to significantly reduce the risk of skeletal complications. N-BPs inhibit farnesyl bisphosphate (FPP) synthase, whose activity is crucial in the mevalonate pathway, by preventing lipid prenylation of small GTPases, such as Ras, Rho and Rac, and, consequently, blocking downstream signaling pathways and inducing apoptosis of osteoclasts and tumor cells [10-13]. Recent data suggested that ZOL, alone or in combination with neoadjuvant chemotherapy, exerts direct or indirect anticancer effects on a variety of cancers, including BC [14-16]. In particular, the in vitro anticancer activity of $\mathrm{ZOL}$ is correlated to reduced migration, invasion, adhesion and proliferation and increased apoptosis of cancer cells [17-20]. ZOL also exhibits indirect anticancer activities, by inhibiting angiogenesis [21] and tumor-associated macrophage infiltration and promoting cytotoxicity of $\gamma \delta$ T cells [22-24].

Using a microarray platform, we previously demonstrated that ZOL could modulate the expression of genes involved in metabolic processes, cytoskeletal and ECM organization, cell communication and cell proliferation pathways in MCF7 BC cell line. Therefore, ZOL has been shown to inhibit the invasiveness processes in cancer cells by modifying their capability to invade tumor microenvironment and thus turning off their metastatic potential [25]. We found also that low doses of ZOL block cellular proliferation, by inhibiting the phosphorylated state of AKT and MAPK proteins, and affect the cytoskeletal reorganization by up-regulating fibronectin-1 (FN1) and actin. Moreover, we observed that ZOL treatment promotes the TGF- $\beta 1 /$ SMADs pathway and mediates the anti-angiogenic potential in MCF7 cells via up-regulation of the thrombospondin-1 (THBS1) expression [26].

MicroRNAs (miRNAs) are a group of non-coding regulatory small RNAs, 20-22 nucleotides in length, which have been shown to regulate several cellular processes such as proliferation, differentiation, apoptosis, cell metabolism and angiogenesis [27]. MiRNAs can inhibit gene expression by recognizing specific binding sites in the $3^{\prime}$ untranslated region (UTR) of target mRNA molecules [28], leading to their degradation, inhibition of their translation, or both $[27,29]$. The dysregulated expression of miRNAs, observed in almost all human malignancies, is involved in several cancer processes, including cell cycle control, angiogenesis, metastasis, apoptosis, invasion, and resistance to hypoxia [30-33]. Different miRNA expression profiles were associated with specific BC pathologic characteristics, such as tumor stage, progesterone and estrogen receptor expression, vascular invasion and proliferation index [34]. Recent studies showed that several miRNAs are involved in bone metastases formation, by interfering with the crucial steps of cancer cell intravasation and tumor invasion, and targeting specific genes implicated in epithelialmesenchymal transition (EMT), survival, invasiveness, motility, osteomimicry and bone remodeling [35, 36]. Many miRNAs were identified as mediators of bone metastases acting as oncomiR (miR-17-92, miR-373, miR-520c) or anti-oncomiR (miR-7, miR-30, miR-34a, miR-143, miR-145, miR-335) in tumor invasion processes [37]. In a recent paper, Croset et al. [38] have grouped the main miRNAs involved in bone metastasis development into three following processes: bone remodeling (miR-33a and miR-326), osteomimicry (miR-30s family, miR-204, miR-211, miR-218 and miR-379) and EMT (Let-7 family, miR-7, miR-10b, miR-34a, miR-100, miR-143, miR-145, miR-200 family, miR-203 and miR-205). Specific miRNAs involved in regulation of $\mathrm{BC}$ bone metastases were found to be up-regulated (miR-10b, miR-21, miR-135a, miR-155, miR-221/222, miR-224, miR-373 and miR-520c) and down-regulated (miR-30s, miR-31, miR-34a, miR-125, miR-200, miR-203, miR-205, miR-206 and miR-342). Five miRNAs of the miRNA-30s family are specifically involved in $\mathrm{BC}$ cell dissemination to bone, by modulating expression of osteomimetic genes such as Runx 2 , connexin 43 , integrin- $\beta 3$, cadherin-11 and CTGF [39].

The data on miRNA expression profiles in human cancer demonstrates that miRNAs are promising predictive, prognostic and/or diagnostic markers [40, 41]. Specific cancer-related miRNA expression patterns could be very helpful to evaluate the efficacy of several drugs in the treatment or prevention of tumors and allow to elucidate underlying molecular mechanisms.

Considering the antitumoral activity of $\mathrm{ZOL}$ in $\mathrm{BC}$ and the important role of miRNAs in regulating different cellular networks, we analyzed the miRNA expression profile in MCF7 BC cell line after treatment with low doses of ZOL to better understand if and how the molecular mechanism by which ZOL mediates these effects involves miRNAs. Only one dose of ZOL was used, because in our previous work [26] three different concentrations $(10,50$ and $100 \mu \mathrm{M})$ at three different time-points $(24,48$ and $72 \mathrm{~h}$ ) were tested, and the lower dose $(10 \mu \mathrm{M})$ and the best time-point $(24 \mathrm{~h})$, sufficient to 
induce an anti-proliferative effect on MCF-7 cells, were identified by in vitro cell viability assays (data not shown). We performed an expression study on a set of 377 human miRNAs and validated the expression of 11 specific ZOL-induced miRNAs in MCF-7 and SkBr3 BC cells. For the first time, we showed a panel of differentially expressed miRNAs in cells treated with ZOL compared to untreated cells and established, at the light of previous our results [26], the correlation between these miRNAs and cancer-related pathways.

\section{RESULTS}

\section{MiRNA expression profile induced by ZOL}

Understanding mechanisms underlying miRNAs function in the cancer onset and progression provides an useful tool to develop different strategies able to use miRNAs as potential targets for cancer treatment. Since several experimental studies reported that miRNAs may be significant diagnostic and prognostic biomarkers in human tumors, alterations in miRNA expression patterns of different cancers can be associated to specific pathological aspects, disease outcome and treatment response. Nowadays, there is no evidence of dysregulated miRNAs in response to ZOL treatment, therefore it would be original and intriguing to identify the molecular mechanisms allowing their use in anticancer therapeutic strategies. In order to understand the effect of lowdose ZOL treatment on miRNA expression profile we performed a large-scale analysis of 377 miRNAs on MCF7 cells treated with $10 \mu \mathrm{M}$ ZOL for $24 \mathrm{~h}$ compared to untreated cells.

Among 377 human miRNAs, about $40 \%$ of them (150) was not detected in MCF-7 cells. Analysis showed 205 microRNAs expressed in MCF7 BC cells after treatment with $10 \mu \mathrm{M}$ ZOL for $24 \mathrm{~h}$ (data not shown). This result suggests that only a part of the miRNA population is expressed in human MCF-7 BC cells. Accumulating evidence showed that several miRNAs are expressed in a tissue- or species specific manner and only a small amount of miRNAs is expressed in a specific tissue at a determined time [42]. Our result is consistent with this conclusion.

In order to highlight the significantly expressed miRNAs we established a cut off of fold change $>2$ for up-regulated miRNAs and $<0.3$ for down-regulated miRNAs. Statistical analysis revealed 54 miRNAs differentially expressed in MCF7 cells treated with $10 \mu \mathrm{M}$ ZOL compared to control cells (Figure 1B). Among these 54 miRNAs, we identified 9 up-regulated miRNAs, 12 down-regulated miRNAs, 11 miRNAs specifically induced by ZOL treatment and 22 silenced miRNAs in MCF7 cells treated with ZOL (Figure 1C).

\section{Functional analysis of miRNAs up- and down- regulated by $\mathrm{ZOL}$ treatment}

In order to investigate the biological role of differentially expressed miRNAs in MCF7 cells treated with $10 \mu \mathrm{M}$ ZOL, we used mirPath software. Nine upregulated and twelve down-regulated miRNAs with different expression fold changes in comparison to untreated cells were found (Figure S1). For statistical analysis only the intersection of targeted genes (hypothetical genes targeted by all selected miRNAs) was evaluated [43].

The obtained results showed the involvement of ZOL in PI3K-Akt signaling pathway, one of the most significant pathways in cancer biology. Statistical analysis showed that 6 ZOL-deregulated miRNAs, such as miR-142-3p, miR-483-5p, miR-486-5p, miR-502-5p, miR-627 and miR-96-5p, shared 60 genes involved in the PI3K-Akt signaling pathway (Table 1). The phosphorylation process of AKT and ERK1/2 can be inhibited by up to $75 \%$ and $36 \%$, respectively, after $24 \mathrm{~h}$ of ZOL treatment [44]. The extent of inhibition of phosphorylated-protein kinase B (p-AKT) and phosphorylated-mammalian target of rapamycin (p-mTOR) was responsible for inhibitory effect of ZOL on cell growth [45]. Moreover, we showed that also lowdose ZOL treatment $(10 \mu \mathrm{M})$ reduces both MAPK and Akt activities, by which ZOL slows the cell proliferation and spread of cancer cells after they have colonized bone [26].

We identified other pathways such as lysine degradation (10 genes, 4 miRNAs), Wnt signaling (22 genes, 5 miRNAs), TGF- $\beta$ signaling (11 genes, 4 miRNAs), Jak-STAT signaling (24 genes, 5 miRNAs), regulation of actin cytoskeleton ( 36 genes, 3 miRNAs) and mTOR signaling (19 genes, 3 miRNAs) (Table 2).

Actin reorganization [26], cell cycle progression [46], apoptosis [47], angiogenesis [48], DNA repair $[49,50]$, NF-kB signaling [51] are elucidated pathways through which ZOL exerts its anticancer activity. Our analysis showed that miRNAs are possible molecular mediators of these effects.

\section{ZOL induces expression of specific miRNAs}

A further series of studies was conducted on the miRNAs expression profile data in order to identify any pathway of particular interest. Array data showed that ZOL treatment induces expression of 11 miRNAs in MCF7 cells. In order to validate miRNA expression, quantitative Real-Time PCR was performed on another pair of independent samples from MCF7 and SkBr3 cells using TaqMan miRNA assays. Data confirmed that 11 miRNAs were specifically expressed in both MCF7 and $\mathrm{SkBr} 3$ cells treated with $10 \mu \mathrm{M}$ ZOL for $24 \mathrm{~h}$ compared to 
untreated cells (Figure 2A). Seven miRNAs were mainly expressed: let-7f, miR-142-5p, miR-302a-3p, miR-326, miR-449b-5p, miR-516b-5p and miR-570-5p (Table S1). Untreated cells showed undetermined values of $\mathrm{Ct}$ for all 11 miRNAs. To understand the biological role of these miRNAs we created heat maps of miRNAs versus pathways using miRPath v2.0.

The integrated analysis was performed on 7 miRNAs induced after ZOL treatment. This analysis indicated that miRNAs showing high expression levels were included in the following three pathways: PI3K/Akt signaling (hsa04151), MAPK signaling (hsa 04010) and regulation of actin cytoskeleton (hsa 04010) (Figure 2B). In particular, we found 5 miRNAs, among the 7 most representative miRNAs, implicated in the regulation of $\mathrm{PI}$ K/Akt signaling pathway, suppressing the expression of 87 hypothetical genes; 5 miRNAs inhibiting 55 potential targets were involved in MAPK signaling cascade; and

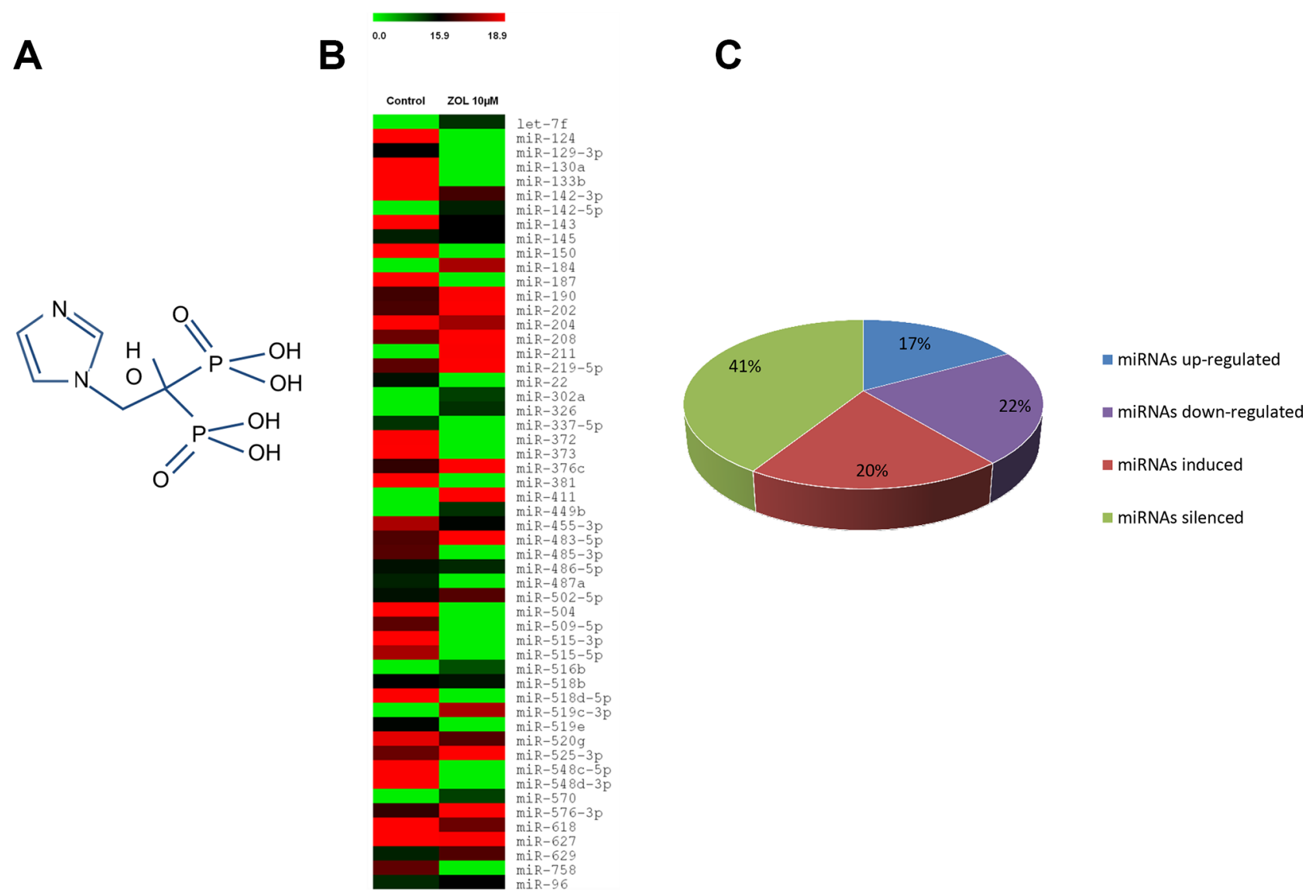

the pathway of actin cytoskeleton regulation was shared by 3 miRNAs modulating 40 potential candidate genes. In addition, the ubiquitin-mediated proteolysis ( 25 genes, 2 miRNAs), mTOR (16 genes, 3 miRNAs), Erb (20 genes, 3 miRNAs), and TGF- $\beta$ (16 genes, 2 miRNAs) signaling pathways and focal adhesion molecules (25 genes, 2 miRNAs) were statistically relevant (Table 3).

\section{ZOL silences the expression of specific miRNAs}

Our results showed that miRNA expression pattern of MCF-7 BC cells was distinctly different between untreated and treated cells with $10 \mu \mathrm{M}$ ZOL for $24 \mathrm{~h}$.

Microarray data indicated that 22 miRNAs were specifically detected in untreated MCF-7 cells, but not in cells exposed to $10 \mu \mathrm{M}$ ZOL for $24 \mathrm{~h}$ (Table S2). These results were confirmed in independent samples from both MCF7 and SkBr3 cells by means of quantitative

Figure 1: MiRNA expression profile induced by ZOL. (A) Chemical structure of zoledronic acid. (B) Heat map of differentially expressed miRNAs by ZOL in breast cancer cells. The heat map was generated from microarray data reflecting expression values in MCF-7 cells treated with $10 \mu \mathrm{M}$ ZOL for $24 \mathrm{~h}$ in comparison to untreated cells (control). Only up-regulated miRNAs with fold change $>2$ and down-regulated miRNAs with fold change $<0.3$ were considered $(P<0.05)$. Each row represents the expression levels for a single miRNA tested for two different experimental conditions. Each column shows the expression levels for the miRNAs tested for a single experimental condition. The absolute expression value of each miRNA is derived from the mean of two biological replicates. The color scale bar on the top represents signal intensity variations ranging from green (poorly expressed or unexpressed miRNAs) to red (highly expressed miRNAs). Black boxes indicate intermediate expression values. (C) Pie chart representation of the 54 differentially expressed miRNAs obtained by miRNAs expression profile: 9 up-regulated miRNAs, 12 down-regulated miRNAs, 11 miRNAs induced by ZOL and 22 silenced miRNAs in MCF7 treated with ZOL. 
Table 1: Hypothetical gene targets of 6 miRNAs deregulated by ZOL in MCF7

\begin{tabular}{|c|c|c|c|}
\hline \multicolumn{4}{|c|}{ PI3K/Akt signaling pathway } \\
\hline PRLR & BCL2 & PIK3R1 & PIK3CA \\
\hline PDGFRA & CDKN1B & JAK3 & CREB3L1 \\
\hline IFNA4 & PPP2R5D & PIK3CG & FOXO3 \\
\hline ITGB8 & GNB1 & FGF9 & IFNA7 \\
\hline NRAS & COL6A6 & IRS1 & FN1 \\
\hline PPP2R3A & KRAS & RAC1 & IFNA17 \\
\hline YWHAE & CDK6 & IFNA16 & FGF23 \\
\hline THBS2 & IL7R & FGF18 & MTOR \\
\hline PIK3R2 & GHR & LAMC1 & ITGA6 \\
\hline PIK3R5 & IKBKB & IGF1 & TNN \\
\hline YWHAG & $\overline{\text { BRCA1 }}$ & BCL2L1 & PTEN \\
\hline CREB1 & ITGAV & PDGFC & MAPK1 \\
\hline$\overline{\text { GNG12 }}$ & GNB2 & CREB3L2 & IFNA10 \\
\hline GNB3 & JAK2 & IFNA14 & FGF7 \\
\hline PIK3AP1 & EIF4E & PDGFD & GRB2 \\
\hline
\end{tabular}

The table represents 60 supposed targets of 6 differentially expressed miRNAs in MCF7 cells after low-dose ZOL treatment. The list was generated by DIANA-miRPath v2.0. $p<0.005$.

Table 2: Cellular pathways modulated by 21 differentially expressed miRNAs in MCF7 cells treated with ZOL

\begin{tabular}{|c|c|c|}
\hline Pathway & miRNAs & Genes \\
\hline PI3K / Akt signaling pathway & 6 & 60 \\
\hline Lysine degradation & 4 & 10 \\
\hline Wnt signaling pathway & 5 & 22 \\
\hline TGF- $\beta$ signaling pathway & 4 & 11 \\
\hline Jak-STAT signaling pathway & 5 & 24 \\
\hline Regulation of actin cytoskeleton & 3 & 36 \\
\hline mTOR signaling pathway & 3 & 19 \\
\hline
\end{tabular}

The pathways were obtained using DIANA-miRPath v2.0. The first column describes the pathway, the middle column reports the number of miRNAs involved in the same pathway and the last column the number of target genes. $p<0.005$.

Real-Time PCR analyses (data not shown). In order to elucidate the biological role of these silenced miRNAs, we created a miRNAs versus pathway heat map (Figure 3).

The integrated analysis was performed on miR-129-3p, miR-130a, miR-133b, miR-150, miR-22, miR-372, miR-373, miR-381, miR-485, miR-504, miR509-5p, miR-515-3p, miR-515-5p, miR-519e, miR548c-5p, miR-548d-3p and miR-758. In this analysis we have not considered miR-124, miR-187, miR-337-5p, miR-487a and miR-518d-5p because they have not been shown to share potential gene targets. The results showed that 10 miRNAs could modulate 95 gene targets involved in MAPK signaling cascade and 9 miRNAs could regulate genes involved in PI3K/Akt signaling pathway (Table 4). The p38-mitogen-activated protein kinase (MAPK) pathway is involved in the mechanism of the antitumor effect of ZOL according to our previous results [26]. ZOL mediated growth inhibition of BC cells in a dose- and time-dependent manner [26], regulated by changes in expression and/or membrane localization of Ras, Rap1, and phosphorylated MAPK [52]. Moreover, we found that 10 miRNAs, modulating 72 potential gene targets, were implicated in the regulation of endocytosis, and 35 genes involved in TGF- $\beta$ signaling pathway could be modulated by 6 miRNAs. In addition, statistically relevant pathways were: Wnt signaling (58 genes, 9 miRNAs), ubiquitinmediated proteolysis (49 genes, 8 miRNAs) and regulation of actin cytoskeleton (74 genes, 8 miRNAs) (Table 4).

\section{DISCUSSION}

Nowadays, although clinicians have several treatment options (chemotherapy, hormone therapy, and targeted therapy), $\mathrm{BC}$ is still responsible for a significant percentage of cancer deaths in women [53,54]. ZOL showed anti-tumoral and anti-metastatic activity during 
cancer progression in preclinical and clinical studies [55]. Several clinical trials analyzed the effects of ZOL on overall survival of $\mathrm{BC}$ patients $[56,57]$. The mechanism by which ZOL explicates its antitumor properties has already been studied, and its inhibitory effect on tumor angiogenesis has been demonstrated. It has been reported that proliferation, migration and invasion are inhibited by $\mathrm{ZOL}$ in order to promote apoptosis and reduce the adhesion to bone of malignant cells $[26,58,59]$.

The main aim of our study was to investigate the molecular mechanisms by which ZOL exerts its antitumoral effects in $\mathrm{BC}$ cells focusing our attention on
miRNAs. Based on the TaqMan Low density array analysis, we identified 54 differentially expressed miRNAs in human MCF-7 BC cells after treatment with $10 \mu \mathrm{M}$ ZOL for $24 \mathrm{~h}$ with different abundance for each miRNA. Most of these miRNAs has not been reported or investigated in BC cells. Our analyses showed that lowdose ZOL treatment affected the expression levels of some miRNAs in MCF-7 cells. In order to identify the cellular pathways modulated by deregulated miRNAs we performed an integrated analysis using mirPath software. MiRNAs versus pathway heat map showed that, among the 21 deregulated miRNAs, 6 miRNAs shared 60 genes

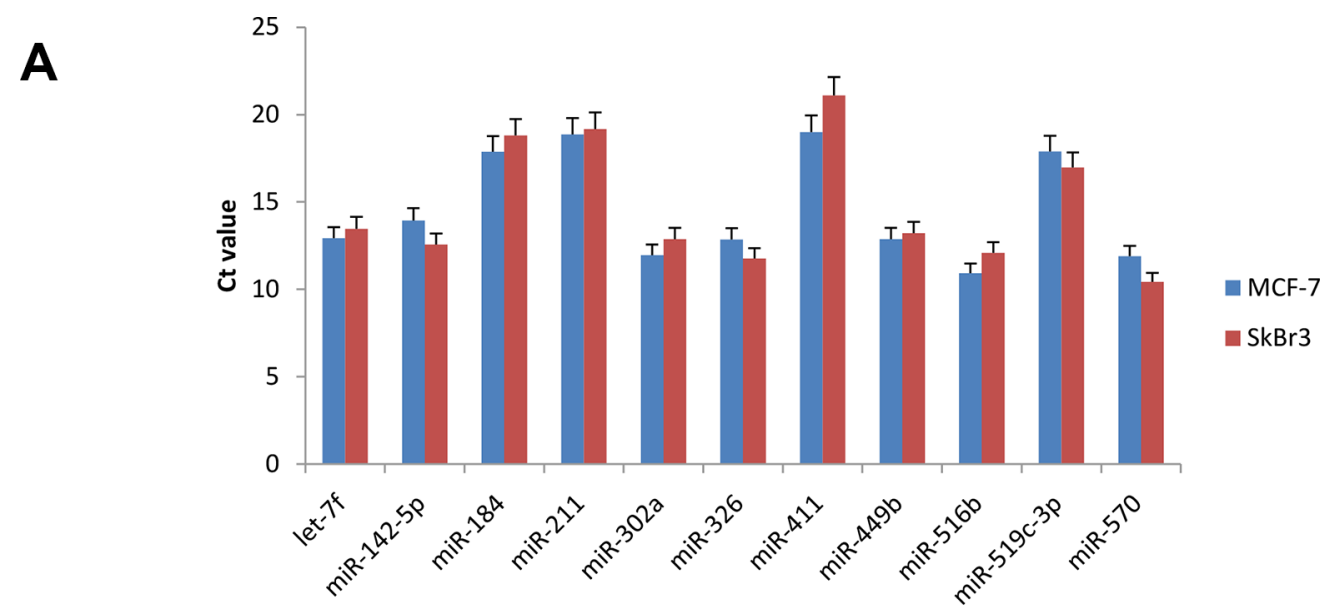

B

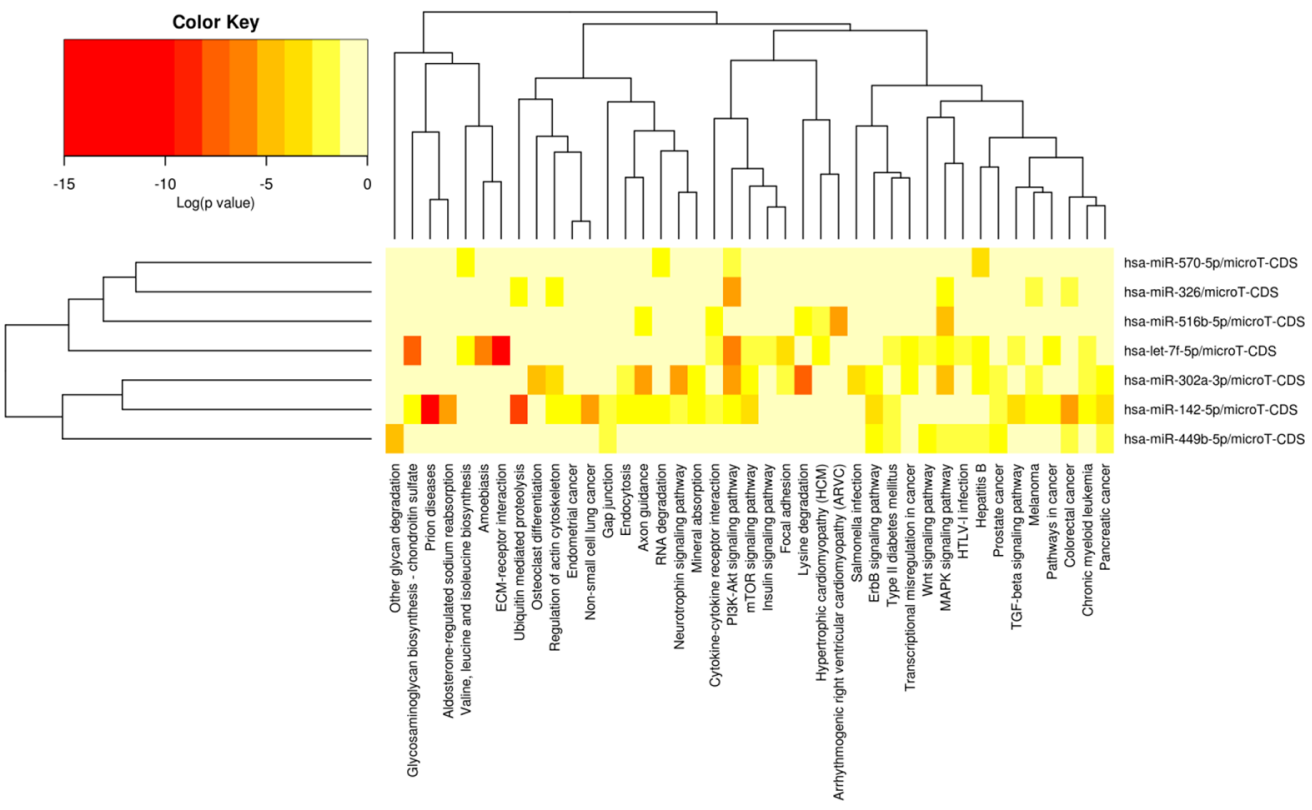

Figure 2: ZOL induces the expression of 11 specific miRNAs. (A) Validation of miRNA array data by quantitative real-time PCR analysis. The mean Ct values of let-7f, miR-142-5p, miR-184, miR-211, miR-302a-3p, miR-326, miR-411, miR-449b-5p, miR-516b-5p, miR-519c-3p and miR-570-5p were determined in MCF-7 and SkBr3 cells treated with $10 \mu \mathrm{M}$ ZOL for 24 h. RNU48 was used as endogenous control. Data are presented as $\mathrm{Ct}$ values \pm SDs. Untreated cells showed undetermined values of $\mathrm{Ct}$ for all $11 \mathrm{miRNAs}$; (B) miRNAs versus pathways heat map (clustering based on significance levels). Darker colors represent lower significance values. The dendrograms placed on both axes depict hierarchical clustering results for miRNAs and pathways, respectively. On the miRNA axis, we can identify clustered miRNAs by exhibiting similar pathway targeting patterns. An analogous clustering can be observed also on the pathway axis. Hierarchical clustering was realized using DIANA-miRPath v2.0. 
Table 3: Cellular pathways modulated by specific miRNAs induced by ZOL in MCF7 and SkBr3 cells

\begin{tabular}{|l|c|c|}
\hline \multicolumn{1}{c}{ Pathway } & miRNAs & Genes \\
\hline PI3K /Akt signaling pathway & 5 & 87 \\
\hline MAPK signaling pathway & 5 & 55 \\
\hline Regulation of actin cytoskeleton & 3 & 40 \\
\hline Ubiquitin mediated proteolysis & 2 & 25 \\
\hline mTOR signaling pathway & 3 & 16 \\
\hline Erb signaling pathway & 3 & 20 \\
\hline Focal adhesion & 2 & 25 \\
\hline TGF- $\beta$ signaling pathway & 2 & 16 \\
\hline
\end{tabular}

The pathways were obtained using DIANA-miRPath v2.0. The first column reports the pathway, the middle column shows the number of miRNAs involved in the same pathway and the last column reports the number of the target genes. $p<0.005$.

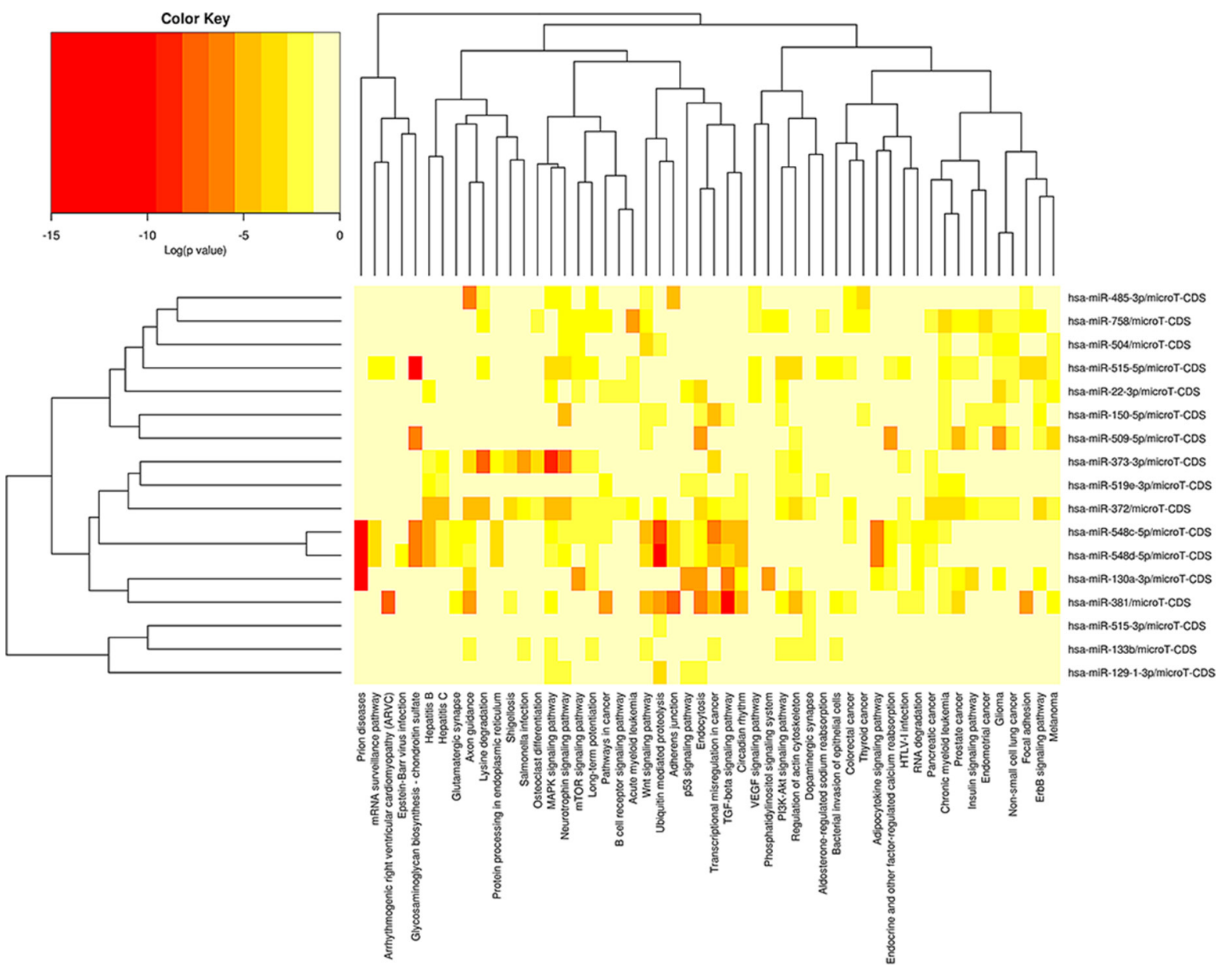

Figure 3: ZOL silences the expression of 22 miRNAs in breast cancer cells. MiRNAs versus pathways heat map (clustering based on significance levels). Darker colors represent lower significance values. The dendrograms placed on both axes depict hierarchical clustering results for miRNAs and pathways, respectively. On the miRNA axis, we can identify clustered miRNAs by exhibiting similar pathway targeting patterns. An analogous clustering can be observed also on the pathway axis. Hierarchical clustering was realized using DIANA-miRPath v2.0. 
Table 4: Cellular pathways modulated by silenced miRNAs in MCF7 and SkBr3 cells treated with ZOL

\begin{tabular}{|c|c|c|}
\hline Pathway & miRNAs & Genes \\
\hline MAPK signaling pathway & 10 & 95 \\
\hline PI3K-Akt signaling pathway & 9 & 106 \\
\hline Endocytosis & 10 & 72 \\
\hline TGF- $\beta$ signaling pathway & 6 & 35 \\
\hline Wnt signaling pathway & 9 & 58 \\
\hline Ubiquitin mediated proteolysis & 8 & 49 \\
\hline Regulation of actin cytoskeleton & 8 & 74 \\
\hline
\end{tabular}

The pathways were obtained using DIANA-miRPath v2.0. The first column reports the pathway, the middle column shows the number of miRNAs involved in the same pathway and the last column reports the number of the target genes. $p<0.005$.

involved in the PI3K-Akt signaling pathway. Lysine degradation, Wnt signaling, TGF- $\beta$ signaling, Jak-STAT signaling, regulation of actin cytoskeleton and mTOR signaling were other significantly relevant pathways identified by statistical analysis. We also found that $10 \mu \mathrm{M}$ ZOL treatment for $24 \mathrm{~h}$ induced expression of 11 specific miRNAs in MCF7 and $\mathrm{SkBr} 3$ cells. We focused our attention on 7 miRNAs which were more expressed than others: let-7f, miR-142-5p, miR-302a-3p, miR-326, miR-449b-5p, miR-516b-5p and miR-570-5p.

The integrated analysis indicated that miRNAs showing high expression levels were involved in PI3K/ Akt signaling, MAPK cascade, regulation of actin cytoskeleton, ubiquitin-mediated proteolysis, mTOR, TGF $-\beta$ and Erb signaling pathways, and focal adhesion molecules.

In contrast, 22 miRNAs were expressed in untreated MCF7 and SkBr3 cells only, indicating that low-dose ZOL treatment silences these miRNAs, probably regulating transcriptional activation factors. Integrated analysis showed that 17 miRNAs could modulate genes involved in MAPK, PI3K/Akt, TGF- $\beta$ and Wnt signaling pathways, ubiquitin-mediated proteolysis, and regulation of actin cytoskeleton. Overall, miRNAs regulated by low doses of ZOL could modulate genes involved in cancer-related pathways.

Additionally, a comparison between the microRNA expression profile described in our work and that obtained in other recent papers concerning the involvement of miRNAs in bone metastasis formation showed that lowdose ZOL treatment up-regulates three miRNAs opposing to metastasis development (miR-143, miR-145 and miR204), induces specific expression of miR-211 (suppressor of osteoclast function), and specifically suppresses the expression of miR-373 (metastasis promoter). Furthermore, our data showed a ZOL-induced downregulation of the miR-96 expression, whose up-regulation, conversely, has been shown to increase cell proliferation in human $\mathrm{BC}$ via direct targeting of $\mathrm{FOXO3a}$ [60].
In addition, our analyses revealed a specific ZOL-mediated induction of expression of miR-302a and miR-326, that is perfectly coherent with the recent findings from Liang and collaborators demonstrating the involvement of these miRNAs in invasion and metastasis, and therapy resistance, respectively, in $\mathrm{BC}[61,62]$. Indeed, the restoration of the miR-302a expression has been shown to inhibit the invasive ability and metastasis both in vitro and in vivo by down-regulation of CXCR4 expression [61], whereas the ectopic expression of miR-326 sensitized multidrug-resistant $\mathrm{BC}$ cells to chemotherapy by downregulation of MRP-1 expression [62]. Lastly, since Pandey et al. [63] recently reported that high expression levels of miR-22 are associated with progression, metastasis and poor prognosis in $\mathrm{BC}$ patients, our results showing the ZOL-mediated silencing of miR-22 expression support the already known role of $\mathrm{ZOL}$ as effective anticancer agent.

Designing therapies targeting the metastasis development mechanisms could prevent the escape of BC cells from a primary tumor and inhibit bone metastasis formation. The current study demonstrates that aberration of specific miRNAs after ZOL treatment may be effector of the anti-tumoral activity of $\mathrm{ZOL}$ in $\mathrm{BC}$ cells.

The microRNA expression profile obtained in this work is correlated with cancer-related biological pathways, such as PI3K/Akt, MAPK, TGF- $\beta$ signaling and actin cytoskeletal remodeling. This data is in agreement with that reported in our previous work [26], where we confirmed, using the same ZOL concentration, the phosphorylation inhibition of the AKT and MAPK proteins. The obtained results are indicative of the mechanisms by which ZOL is able to inhibit cellular proliferation [26]. Also, we found that ZOL plays an inhibitory role in $\mathrm{BC}$ cell invasion through cytoskeletal remodeling. The molecular mechanism underlying this effect is the activation of TGF- $\beta 1 /$ Smad signaling pathway and downstream activity of FN1 and $\beta$-actin. In future, investigating the molecular regulatory mechanism of drugspecific miRNAs will allow a better understanding of the 
action mechanism of ZOL and the detection of novel potential targets useful for the development of possible new therapeutic strategies.

\section{MATERIALS AND METHODS}

\section{Cell culture}

Human BC cell lines, MCF-7 and SkBr3, purchased from the American Type Culture Collection (Rockville, MD, USA) were grown in Dulbecco's modified Eagle's medium Gibco DMEM:F12 (Invitrogen, Carlsbad, CA,USA) containing 10\% fetal bovine serum (FBS) and $1 \%$ Penicillin/Streptomycin (P/S) (Gibco). Cells were incubated at $37^{\circ} \mathrm{C}$ in a humidified atmosphere of $5 \%$ of $\mathrm{CO}_{2}$. Eighty per cent confluent cultures were stimulated with ZOL $10 \mu \mathrm{M}$ for $24 \mathrm{~h}$. ZOL was kindly provided by Novartis Pharma AG.

\section{MiRNA expression profile analysis}

Total cellular RNA and miRNAs has been isolated using the miRNeasy Mini Kit (Qiagen Inc, Valencia, CA). The quality of the samples have been controlled through RNA 6000 Nano Assay (Agilent Techologies, Palo Alto, CA, USA) using 2100 Bioanalyzer (Agilent Technologies, Santa Clara, CA) and quantified through the spectrophotometer NanoDrop ND-1000 (CELBIO). To study miRNA expression profile, we used TaqMan ${ }^{\circledR}$ Low Density Array A Human MicroRNA v2.0 (Life Technologies, Carlsbad, California, U.S.). The arrays were performed in accordance to manufacturer's protocols as previously described [64]. Briefly, $600 \mathrm{ng}$ of miRNAenriched total RNA were reverse transcribed using Megaplex $^{\text {TM }}$ RT Primers Human Pool A (Life Technologies, Carlsbad, California, U.S.) according to manufacturer's instructions. Conditions for the reverse transcription reaction were as follows: $16^{\circ} \mathrm{C}$ for 2 minutes, $42^{\circ} \mathrm{C}$ for 1 minute, $50^{\circ} \mathrm{C}$ for 1 second for 40 cycles, $85^{\circ} \mathrm{C}$ for 5 minutes then hold at $4^{\circ} \mathrm{C}$. Obtained cDNA was diluted, mixed with TaqMan Gene Expression Master Mix, and loaded into each of the eight fill ports on the TaqMan ${ }^{\circledR}$ Human MicroRNA Array A (Life Technologies, Carlsbad, California, U.S.). The TaqMan Human MicroRNA Array is a 384-well microfluidics card containing 377 primer-probe sets for individual miRNAs as well as three carefully selected candidate endogenous small nucleolar RNAs control assay and one negative control assay. The array was centrifuged at 1,200 rpm twice for 1 minute each, then run on ABI-PRISM 7900 HT Sequence Detection System (Applied Biosystems). Two biological replicates were performed for each experimental condition. The data were quantified using the SDS 2.4 software and normalized using the RNU48 as endogenous control. The cycle threshold $(\mathrm{Ct})$ value, which was calculated relatively to the endogenous control, was used for our analysis $(\Delta \mathrm{Ct})$. The $2^{-\triangle \Delta C T}$ (delta-delta-Ct algorithm) method was used to calculate the relative changes in miRNA expression. A miRNA was defined differentially expressed when estimated $P$-value was $<0.05$.

\section{Quantitative real-time PCR}

Ten nanograms of total RNA from another independent experiment were reverse transcribed using Taqman MicroRNA Reverse Transcription Kit (Life Technologies, Carlsbad, California, U.S.) according to manufacturer's instructions. The obtained cDNA was amplified using the following Taqman MicroRNA assays: hsa-let-7f-5p, hsa-miR-142-5p, hsa-miR-184, hsa-miR-211, hsa-miR-302a, hsa-miR-326, hsa-miR-411, hsa-miR-449b, hsa-miR-516b, hsa-miR-519c-3p and hsamiR-570 (Life Technologies, Carlsbad, California, U.S.). To normalize quantitative Real-Time PCR reactions, parallel reactions were run on each sample for RNU48 snRNA. The reactions were performed in triplicate and changes in the target miRNA content relative to RNU6B were determined using the comparative $\mathrm{Ct}$ method to calculate changes in $\mathrm{Ct}$, and, ultimately, fold and percent change. An average $\mathrm{Ct}$ value for each RNA was obtained for replicate reactions.

\section{MiRNA data analysis}

Hierarchical cluster and heat map analyses were performed using the MultiExperiment Viewer (MeV v4.8) program of TM4 Microarray Software Suite. Heat maps of miRNAs versus pathways were generated using miRPath v2.0 database as previously described [43, 65]. DIANA-miRPath v2.0 is based on a new relational schema, specifically designed to accommodate this as well as future miRPath updates. MiRNA and pathway related information was obtained from miRBase 18 [66] and Kyoto Encyclopedia of Genes and Genomes (KEGG) v58.1 [67]. Hierarchical clustering of targeted pathways and miRNAs was realized using DIANA-miRPath v2.0. The software created a clustering of the selected miRNAs based on their influence on molecular pathways [65].

\section{Statistical analysis}

Filtering criteria able to select reliably quantifiable miRNAs were used (cut off $<35 \mathrm{Ct}$ ). Undetermined values of $\mathrm{Ct}$ were estimated as $40 \mathrm{Ct}$ (the last cycle of the reactions). Heat maps were constructed using z-transformed relative gene $\Delta \mathrm{Ct}$ values, so that measurements were scaled to obtain gene-wise zero mean and unit variance. Data are represented as mean \pm S.D (standard deviation). Statistical analyses were performed by Student's $t$-test. Values of $P<0.05$ were considered to be statistically significant. 


\section{ACKNOWLEDGMENTS}

Dr. Chiara Drago contributed to the revision of the English language of this manuscript.

\section{CONFLICTS OF INTEREST}

The authors declare no conflicts of interest.

\section{GRANT SUPPORT}

This work was supported by the Fondazione Mediterranea "G.B. Morgagni”, Catania, Italy.

\section{REFERENCES}

1. Fanale D, Bazan V, Caruso S, Castiglia M, Bronte G, Rolfo C, Cicero G, Russo A. Hypoxia and human genome stability: downregulation of BRCA2 expression in breast cancer cell lines. Biomed Res Int. 2013; 2013:746858.

2. Russo A, Calo V, Bruno L, Schiro V, Agnese V, Cascio S, Foddai E, Fanale D, Rizzo S, Di Gaudio F, Gulotta E, Surmacz E, Di Fede G, et al. Is BRCA1-5083del19, identified in breast cancer patients of Sicilian origin, a Calabrian founder mutation? Breast cancer research and treatment. 2009; 113:67-70.

3. Aft R. Protection of bone in premenopausal women with breast cancer: focus on zoledronic acid. Int $\mathrm{J}$ Womens Health. 2012; 4:569-576.

4. Mundy GR. Metastasis to bone: causes, consequences and therapeutic opportunities. Nat Rev Cancer. 2002; 2:584-593.

5. Zhou WB, Zhang PL, Liu XA, Yang T, He W. Innegligible musculoskeletal disorders caused by zoledronic acid in adjuvant breast cancer treatment: a meta-analysis. Journal of experimental \& clinical cancer research. 2011; 30:72.

6. Iozzo RV, Karamanos N. Proteoglycans in health and disease: emerging concepts and future directions. FEBS J. 2010; 277:3863.

7. Syggelos SA, Aletras AJ, Smirlaki I, Skandalis SS Extracellular matrix degradation and tissue remodeling in periprosthetic loosening and osteolysis: focus on matrix metalloproteinases, their endogenous tissue inhibitors, and the proteasome. Biomed Res Int. 2013; 2013:230805.

8. Coleman RE, Lipton A, Roodman GD, Guise TA, Boyce BF, Brufsky AM, Clezardin P, Croucher PI, Gralow JR, Hadji P, Holen I, Mundy GR, Smith MR, et al. Metastasis and bone loss: advancing treatment and prevention. Cancer Treat Rev. 2010; 36:615-620.

9. Facchini G, Caraglia M, Santini D, Nasti G, Ottaiano A, Striano S, Maiolino P, Ruberto M, Fiore F, Tonini G, Budillon A, Iaffaioli RV, Zeppetella GL. The clinical response on bone metastasis from breast and lung cancer during treatment with zoledronic acid is inversely correlated to skeletal related events (SRE). Journal of experimental \& clinical cancer research : CR. 2007; 26:307-312.

10. Body JJ, Bartl R, Burckhardt P, Delmas PD, Diel IJ, Fleisch H, Kanis JA, Kyle RA, Mundy GR, Paterson AH, Rubens RD. Current use of bisphosphonates in oncology. International Bone and Cancer Study Group. J Clin Oncol. 1998; 16:3890-3899.

11. Luckman SP, Hughes DE, Coxon FP, Graham R, Russell G, Rogers MJ. Nitrogen-containing bisphosphonates inhibit the mevalonate pathway and prevent post-translational prenylation of GTP-binding proteins, including Ras. J Bone Miner Res. 1998; 13:581-589.

12. Kopecka J, Porto S, Lusa S, Gazzano E, Salzano G, Giordano A, Desiderio V, Ghigo D, Caraglia M, De Rosa G, Riganti C. Self-assembling nanoparticles encapsulating zoledronic acid revert multidrug resistance in cancer cells. Oncotarget. 2015; 6:31461-31478. doi: 10.18632/ oncotarget.5058.

13. Coxon FP, Rogers MJ. The role of prenylated small GTPbinding proteins in the regulation of osteoclast function. Calcif Tissue Int. 2003; 72:80-84.

14. Morgan G, Lipton A. Antitumor effects and anticancer applications of bisphosphonates. Semin Oncol. 2010; 37 Suppl 2:S30-40.

15. Coleman RE, Winter MC, Cameron D, Bell R, Dodwell D, Keane MM, Gil M, Ritchie D, Passos-Coelho JL, Wheatley D, Burkinshaw R, Marshall SJ, Thorpe H. The effects of adding zoledronic acid to neoadjuvant chemotherapy on tumour response: exploratory evidence for direct anti-tumour activity in breast cancer. Br J Cancer. 2010; 102:1099-1105.

16. Zarogoulidis K, Boutsikou E, Zarogoulidis P, EleftheriadouE, Kontakiotis T, Lithoxopoulou H, Tzanakakis G, Kanakis I, Karamanos NK. The impact of zoledronic acid therapy in survival of lung cancer patients with bone metastasis. Int J Cancer. 2009; 125:1705-1709.

17. Dedes PG, Gialeli C, Tsonis AI, Kanakis I, Theocharis AD, Kletsas D, Tzanakakis GN, Karamanos NK. Expression of matrix macromolecules and functional properties of breast cancer cells are modulated by the bisphosphonate zoledronic acid. Biochim Biophys Acta. 2012; 1820:1926-1939.

18. Sewing L, Steinberg F, Schmidt H, Goke R. The bisphosphonate zoledronic acid inhibits the growth of HCT116 colon carcinoma cells and induces tumor cell apoptosis. Apoptosis. 2008; 13:782-789.

19. Mani J, Vallo S, Barth K, Makarevic J, Juengel E, Bartsch G, Wiesner C, Haferkamp A, Blaheta RA. Zoledronic acid influences growth, migration and invasive activity of prostate cancer cells in vitro. Prostate Cancer Prostatic Dis. 2012; 15:250-255.

20. Ibrahim T, Mercatali L, Sacanna E, Tesei A, Carloni S, Ulivi P, Liverani C, Fabbri F, Zanoni M, Zoli W, Amadori D. Inhibition of breast cancer cell proliferation in repeated and 
non-repeated treatment with zoledronic acid. Cancer Cell Int. 2012; 12:48.

21. Misso G, Porru M, Stoppacciaro A, Castellano M, De Cicco F, Leonetti C, Santini D, Caraglia M. Evaluation of the in vitro and in vivo antiangiogenic effects of denosumab and zoledronic acid. Cancer Biol Ther. 2012; 13:1491-1500.

22. Hamilton E, Clay TM, Blackwell KL. New perspectives on zoledronic acid in breast cancer: potential augmentation of anticancer immune response. Cancer Invest. 2011; 29:533-541.

23. Nussbaumer O, Gruenbacher G, Gander H, Thurnher M. DC-like cell-dependent activation of human natural killer cells by the bisphosphonate zoledronic acid is regulated by gammadelta T lymphocytes. Blood. 2011; 118:2743-2751.

24. Rietkotter E, Menck K, Bleckmann A, Farhat K, Schaffrinski M, Schulz M, Hanisch UK, Binder C, Pukrop T. Zoledronic acid inhibits macrophage/microglia-assisted breast cancer cell invasion. Oncotarget. 2013; 4:1449-1460. doi: 10.18632/oncotarget.1201.

25. Nienhuis HH, Arjaans M, Timmer-Bosscha H, de Vries EG, Schroder CP. Human stromal cells are required for an antibreast cancer effect of zoledronic acid. Oncotarget. 2015; 6:24436-24447. doi: 10.18632/oncotarget.4421.

26. Insalaco L, Di Gaudio F, Terrasi M, Amodeo V, Caruso S, Corsini LR, Fanale D, Margarese N, Santini D, Bazan V, Russo A. Analysis of molecular mechanisms and antitumoural effects of zoledronic acid in breast cancer cells. J Cell Mol Med. 2012; 16:2186-2195.

27. Bartel DP. MicroRNAs: target recognition and regulatory functions. Cell. 2009; 136:215-233.

28. Ma R, Jiang T, Kang X. Circulating microRNAs in cancer: origin, function and application. Journal of experimental \& clinical cancer research. 2012; 31:38.

29. Ambros V. The functions of animal microRNAs. Nature. 2004; 431:350-355.

30. Amodeo V, Bazan V, Fanale D, Insalaco L, Caruso S, Cicero G, Bronte G, Rolfo C, Santini D, Russo A. Effects of antimiR-182 on TSP-1 expression in human colon cancer cells: there is a sense in antisense? Expert Opin Ther Targets. 2013; 17:1249-1261.

31. Wang C, Zheng X, Shen C, Shi Y. MicroRNA-203 suppresses cell proliferation and migration by targeting BIRC5 and LASP1 in human triple-negative breast cancer cells. Journal of experimental \& clinical cancer research. 2012; 31:58.

32. Malek E, Jagannathan S, Driscoll JJ. Correlation of long non-coding RNA expression with metastasis, drug resistance and clinical outcome in cancer. Oncotarget. 2014; 5:8027-8038. doi: 10.18632/oncotarget.2469.

33. Caruso S, Bazan V, Rolfo C, Insalaco L, Fanale D, Bronte G, Corsini LR, Rizzo S, Cicero G, Russo A. MicroRNAs in colorectal cancer stem cells: new regulators of cancer stemness? Oncogenesis. 2012; 1:e32.
34. Iorio MV, Ferracin M, Liu CG, Veronese A, Spizzo R, Sabbioni S, Magri E, Pedriali M, Fabbri M, Campiglio M, Menard S, Palazzo JP, Rosenberg A, et al. MicroRNA gene expression deregulation in human breast cancer. Cancer Res. 2005; 65:7065-7070.

35. Ma L, Weinberg RA. Micromanagers of malignancy: role of microRNAs in regulating metastasis. Trends in genetics: TIG. 2008; 24:448-456.

36. Rahim F, Hajizamani S, Mortaz E, Ahmadzadeh A, Shahjahani M, Shahrabi S, Saki N. Molecular regulation of bone marrow metastasis in prostate and breast cancer. Bone marrow research. 2014; 2014:405920.

37. Santini D, Vincenzi B, Pantano F, Tonini G, Bertoldo F. Targeting bone metastases starting from the preneoplastic niche: home sweet home. Breast cancer research : BCR. 2011; $13: 111$.

38. Croset M, Santini D, Iuliani M, Fioramonti M, Zoccoli A, Vincenzi B, Tonini G, Pantano F. MicroRNAs and bone metastasis: a new challenge. Molecules. 2014; 19:10115-10128.

39. Vimalraj S, Miranda PJ, Ramyakrishna B, Selvamurugan N. Regulation of breast cancer and bone metastasis by microRNAs. Disease markers. 2013; 35:369-387.

40. Visone R, Croce CM. MiRNAs and cancer. Am J Pathol. 2009; 174:1131-1138.

41. Corsini LR, Bronte G, Terrasi M, Amodeo V, Fanale D, Fiorentino E, Cicero G, Bazan V, Russo A. The role of microRNAs in cancer: diagnostic and prognostic biomarkers and targets of therapies. Expert Opin Ther Targets. 2012; 16 Suppl 2:S103-109.

42. Ludwig N, Leidinger P, Becker K, Backes C, Fehlmann T, Pallasch C, Rheinheimer S, Meder B, Stahler C, Meese E, Keller A. Distribution of miRNA expression across human tissues. Nucleic Acids Res. 2016.

43. Papadopoulos GL, Alexiou P, Maragkakis M, Reczko M, Hatzigeorgiou AG. DIANA-mirPath: Integrating human and mouse microRNAs in pathways. Bioinformatics. 2009; 25:1991-1993.

44. Li S, De Souza P. Ras Isoprenylation and pAkt Inhibition by Zoledronic Acid and Fluvastatin Enhances Paclitaxel Activity in T24 Bladder Cancer Cells. Cancers (Basel). 2011; 3:662-674.

45. Lan YC, Chang CL, Sung MT, Yin PH, Hsu CC, Wang KC, Lee HC, Tseng LM, Chi CW. Zoledronic acid-induced cytotoxicity through endoplasmic reticulum stress triggered REDD1-mTOR pathway in breast cancer cells. Anticancer Res. 2013; 33:3807-3814.

46. Chang J, Wang W, Zhang H, Hu Y, Yin Z. Bisphosphonates regulate cell proliferation, apoptosis and pro-osteoclastic expression in MG-63 human osteosarcoma cells. Oncol Lett. 2012; 4:299-304.

47. Kaiser T, Teufel I, Geiger K, Vater Y, Aicher WK, Klein G, Fehm T. Bisphosphonates modulate vital functions 
of human osteoblasts and affect their interactions with breast cancer cells. Breast cancer research and treatment. 2013; 140:35-48.

48. Vincenzi B, Santini D, Dicuonzo G, Battistoni F, Gavasci M, La Cesa A, Grilli C, Virzi V, Gasparro S, Rocci L, Tonini G. Zoledronic acid-related angiogenesis modifications and survival in advanced breast cancer patients. J Interferon Cytokine Res. 2005; 25:144-151.

49. Ory B, Blanchard F, Battaglia S, Gouin F, Redini F, Heymann D. Zoledronic acid activates the DNA S-phase checkpoint and induces osteosarcoma cell death characterized by apoptosis-inducing factor and endonuclease- $G$ translocation independently of p53 and retinoblastoma status. Mol Pharmacol. 2007; 71:333-343.

50. Iguchi T, Miyakawa Y, Saito K, Nakabayashi C, Nakanishi M, Saya H, Ikeda Y, Kizaki M. Zoledronate-induced S phase arrest and apoptosis accompanied by DNA damage and activation of the ATM/Chk1/cdc25 pathway in human osteosarcoma cells. Int J Oncol. 2007; 31:285-291.

51. Schech AJ, Kazi AA, Gilani RA, Brodie AH. Zoledronic acid reverses the epithelial-mesenchymal transition and inhibits self-renewal of breast cancer cells through inactivation of NF-kappaB. Mol Cancer Ther. 2013; 12:1356-1366.

52. Nogawa M, Yuasa T, Kimura S, Kuroda J, Segawa H, Sato K, Yokota A, Koizumi M, Maekawa T. Zoledronic acid mediates Ras-independent growth inhibition of prostate cancer cells. Oncol Res. 2005; 15:1-9.

53. Fanale D, Bazan V, Corsini LR, Caruso S, Insalaco L, Castiglia M, Cicero G, Bronte G, Russo A. HIF-1 is involved in the negative regulation of AURKA expression in breast cancer cell lines under hypoxic conditions. Breast cancer research and treatment. 2013; 140:505-517.

54. Fanale D, Amodeo V, Corsini LR, Rizzo S, Bazan V, Russo A. Breast cancer genome-wide association studies: there is strength in numbers. Oncogene. 2012; 31:2121-2128.

55. Jia XH, Du Y, Mao D, Wang ZL, He ZQ, Qiu JD, Ma XB, Shang WT, Ding D, Tian J. Zoledronic acid prevents the tumor-promoting effects of mesenchymal stem cells via MCP-1 dependent recruitment of macrophages. Oncotarget. 2015; 6:26018-26028. doi: 10.18632/oncotarget.4658.

56. Clezardin P. Mechanisms of action of bisphosphonates in oncology: a scientific concept evolving from antiresorptive to anticancer activities. Bonekey Rep. 2013; 2:267.

57. Jia X, Hong Q, Lei L, Li D, Li J, Mo M, Wang Y, Shao Z, Shen Z, Cheng J, Liu G. Basal and therapy-driven hypoxiainducible factor-1alpha confers resistance to endocrine therapy in estrogen receptor-positive breast cancer. Oncotarget. 2015; 6:8648-8662. doi: 10.18632/oncotarget.3257.
58. Almubarak H, Jones A, Chaisuparat R, Zhang M, Meiller TF, Scheper MA. Zoledronic acid directly suppresses cell proliferation and induces apoptosis in highly tumorigenic prostate and breast cancers. J Carcinog. 2011; 10:2.

59. Li XY, Lin YC, Huang WL, Hong CQ, Chen JY, You YJ, Li WB. Zoledronic acid inhibits proliferation and impairs migration and invasion through downregulating VEGF and MMPs expression in human nasopharyngeal carcinoma cells. Med Oncol. 2012; 29:714-720.

60. Lin H, Dai T, Xiong H, Zhao X, Chen X, Yu C, Li J, Wang X, Song L. Unregulated miR-96 induces cell proliferation in human breast cancer by downregulating transcriptional factor FOXO3a. PLoS One. 2010; 5:e15797.

61. Liang Z, Bian X, Shim H. Inhibition of breast cancer metastasis with microRNA-302a by downregulation of CXCR4 expression. Breast cancer research and treatment. $2014 ; 146: 535-542$.

62. Liang Z, Wu H, Xia J, Li Y, Zhang Y, Huang K, Wagar N, Yoon Y, Cho HT, Scala S, Shim H. Involvement of miR326 in chemotherapy resistance of breast cancer through modulating expression of multidrug resistance-associated protein 1. Biochem Pharmacol. 2010; 79:817-824.

63. Pandey AK, Zhang Y, Zhang S, Li Y, Tucker-Kellogg G, Yang H, Jha S. TIP60-miR-22 axis as a prognostic marker of breast cancer progression. Oncotarget. 2015; 6:4129041306. doi: 10.18632/oncotarget.5636.

64. Di Fiore R, Fanale D, Drago-Ferrante R, Chiaradonna F, Giuliano M, De Blasio A, Amodeo V, Corsini LR, Bazan V, Tesoriere G, Vento R, Russo A. Genetic and molecular characterization of the human osteosarcoma 3AB-OS cancer stem cell line: a possible model for studying osteosarcoma origin and stemness. J Cell Physiol. 2013; 228:1189-1201.

65. Vlachos IS, Kostoulas N, Vergoulis T, Georgakilas G, Reczko M, Maragkakis M, Paraskevopoulou MD, Prionidis K, Dalamagas T, Hatzigeorgiou AG. DIANA miRPath v.2.0: investigating the combinatorial effect of microRNAs in pathways. Nucleic Acids Res. 2012; 40:W498-504.

66. Kozomara A, Griffiths-Jones S. miRBase: integrating microRNA annotation and deep-sequencing data. Nucleic Acids Res. 2011; 39:D152-157.

67. Kanehisa M, Goto S, Sato Y, Furumichi M, Tanabe M. KEGG for integration and interpretation of large-scale molecular data sets. Nucleic Acids Res. 2012; 40:D109-114. 